\title{
Digitizing Traditional Lao Textile to Modern Weave Technique
}

\author{
Lathsamy Chidtavong \\ National University of Laos (NUOL), Vientiane Capital, Laos \\ Michael Winckler, Hans Georg Bock \\ Heidelberg University, Heidelberg, Germany \\ Marion Ellwanger-Mohr \\ University of Applied Sciences, Mönchengladbach, Germany
}

\begin{abstract}
Traditional Lao textiles are wealth in religious motifs, the motifs and patterns on the textiles reflect traditions, beliefs and livelihood of people. The structure of Lao motifs and patterns are complicated, but weaving processes still use traditional techniques and simple floor-loom. Therefore, it takes a lot of time for making a weave-draft on the loom and percentage of losing weave-drafts is very high. In contrast, industrial textiles use electronic loom and digital weave-drafts to produce fabrics, which are suitable for fast production but lack complicated traditional patterns. As a result, this paper introduces scientific approaches for digitizing motifs, patterns, and weave-drafts of traditional Lao textiles. Mathematical principles of Frieze and Wallpaper groups are investigated for digital design. The paper presents two standard files, image file format and WIF file as digital weave-draft in order to fill the gap between traditional and modern weave techniques. The standard files are understandable and usable for both hand-weavers and weaving machines. Our study shows that a modern electronic TC2 loom is a suitable loom to connect between traditional Lao weave technique and modern weave technique.
\end{abstract}

Keywords: frieze group, wallpaper group, Lao textile, WIF file and TC2 loom

\section{Introduction}

Laos consists of a variety of ethnic groups that are rich in traditions and cultures. Traditional Lao textiles are a women-craft heritage on clothes which are rich in religious motifs. The structure of textiles and their elements are a method to express the characteristics of the textile and the difficulties on weaving processes. In Laos, hand ${ }^{1}$ weaving textile is a tradition for self-sufficiency and it provides a small income for women who have little education or who live in rural areas. There are some textile manufacturers in the capital and urban areas, but textile production still uses human labour and most employed weavers are from rural areas. In fact, Lao weavers are skilful at weaving and natural dyeing, but their weaving processes are old tradition, which

Lathsamy Chidtavong, Ph.D., lecturer at Computer Science Department, Faculty of Natural Science, National University of Laos, Vientiane Capital, Lao PDR.

Michael Winckler, Ph.D., Interdisciplinary Center for Scientific Computing (IWR), Heidelberg University, Heidelberg, Germany.

Hans Georg Bock, professor at Interdisciplinary Center for Scientific Computing (IWR), Heidelberg University, Heidelberg, Germany.

Marion Ellwanger-Mohr, professor at Faculty of Textile and Clothing Technology, University of Applied Sciences, Mönchengladbach, Germany.

Correspondence concerning this article should be addressed to Lathsamy Chidtavong, Computer Science Department, Faculty of Natural Science, National University of Laos, P.O. Box:7322, DongDok Campus, Vientiane Capital, Lao PDR. 
contains many technical limitations when compared to modern weave techniques.

The first limitation is that the weavers have no design software and electronic tools for their support, a wooden floor loom is only their main tool. A main weave technique is called "Tam Chok" represented a supplementary weft weave where a master pattern and a weave-draft are set up on the loom by using a traditional method, tying supplementary heddles on the loom. The most risky of this weaving tradition is highly to lose weave-drafts. Another problem arises in this traditional technique; if the weaver wants to set up a new weave-draft there are only two options. The first option is to remove the old draft from the heddles, and then set up a new draft, by this way the weaver can weave a new pattern without waiting until the weaving reaches the end part of the warp threads. The second option is to use another set of heddles, if the weaver intends to reuse this current weave-draft, but the weaver has to wait until finishing the warp threads on the loom. These two choices are very inconvenient, because setting up a weave-draft on the floor-loom is very time consuming. These technical restrictions together with the complexity in motifs and patterns, are still waiting for suitable solutions for connecting to modern weave techniques.

In textile industry however, electronic looms are necessary tools for industrial textiles, especially in developed countries. Weaving processes are controlled by electronic equipment, patterns and weave-drafts are digital files, which can directly be read by electronic looms. The study found that digital weave-drafts are a key to connect traditional Lao weaving techniques and modern weaving technologies, this connection is a first step to introducing a new technical tool to a society of hand weaving textile in Laos, it will help to improve the weaving process of local weavers. Therefore, scientific methods are investigated for generating digital motifs and patterns of Lao textile by using symmetric structure of Frieze and Wallpaper groups. This paper also presented two standards weave file which represent digital weave-draft which are image and WIF files. To accomplish this study three field trips from the North to the South of Laos are conducted for collecting sample of ethnic textiles and using them as a reference for pattern analysis and generation that will be explained below.

\section{Pattern Analysis and Generation}

To analyze stripe and two directional patterns on Lao textiles, the geometric principles of seven symmetry groups in Frieze group and 12 of 17 symmetry groups in wallpaper group (Liu \& Collins, 1998; Radaelli, 2011) are applied. The notation for each group uses international notation from crystallographic groups (IUC: International Union of Crystallography) (Schattschneider, 1978). Moreover, symmetry groups of Frieze group use names that are given by John Conway. In general Frieze and wallpaper patterns are generated by a combination of four basics symmetry operations namely: translation, rotation, reflection, and glide reflection. Frieze patterns are generated by repetitive translation a motif in one direction while wallpaper patterns are generated by repetitive translation a motif in two directions. Therefore, symmetry groups on the textiles are explored by studying symmetric structures of the patterns, then diagrams are constructed where is based on the symmetric structures of each group. The constructed diagrams are used as guidelines for checking patterns' structure and classifying their belonging symmetry group.

\section{Frieze Pattern Analysis and Generation}

The diagram for checking frieze groups illustrates in Figure 1, it helps to check a combination of symmetry operations among the groups. Actually, the seven symmetry groups of Frieze group can be applied for woven pattern, but the pattern style on traditional Lao textiles is related to tradition and religious beliefs of local people. Thus, there are some symmetry groups of Frieze groups which are not found on the Lao textiles. 
The analysis result of Frieze patterns on traditional Lao textiles illustrated that six of seven Frieze groups are found on the textile's samples, they are Spinning Jump (p2mm), Jump (p11m), Sidle (p1m1), Hop (p1), Spinning Hop (p2), and Spinning Sidle (p2mg).

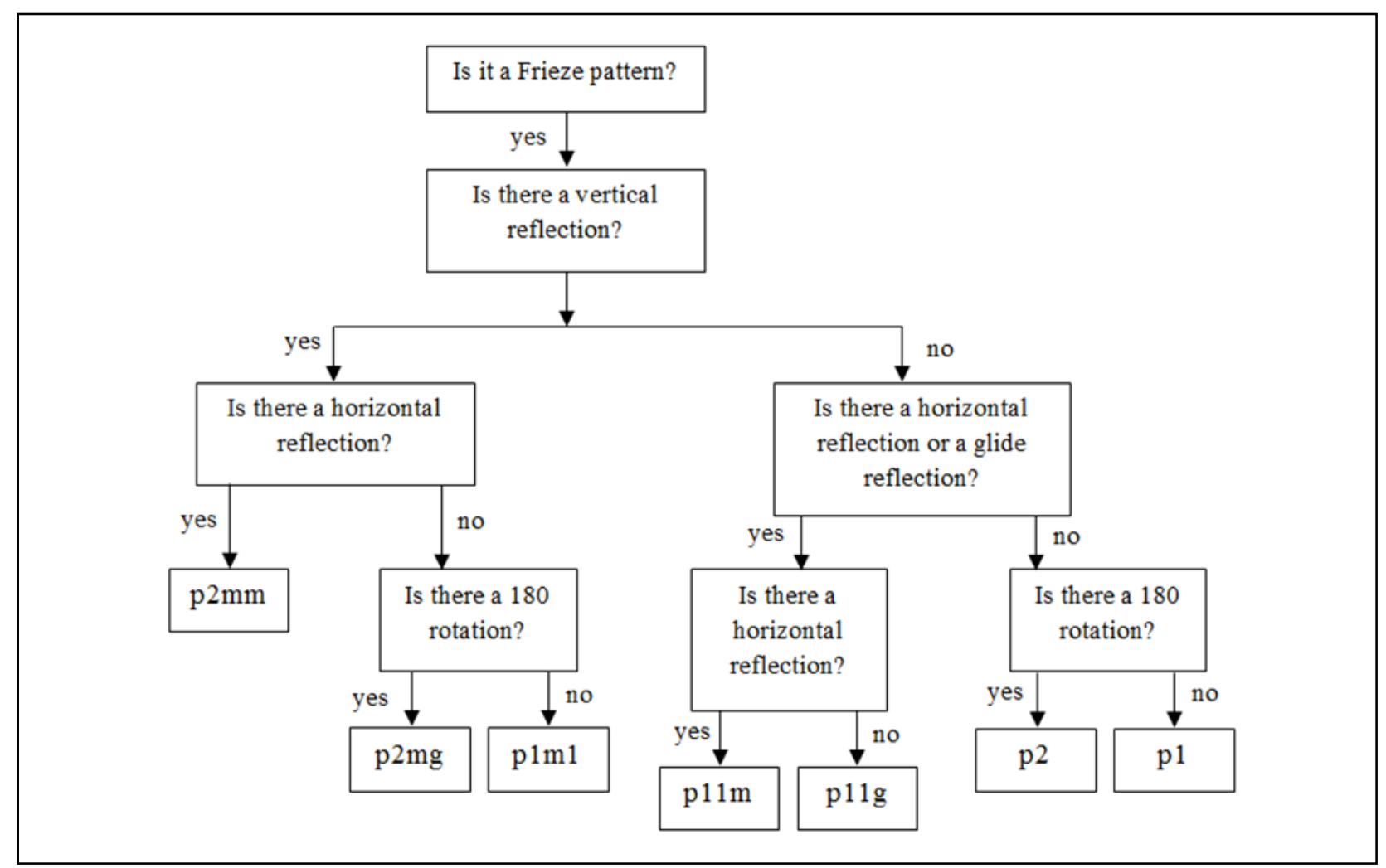

Figure 1. A diagram for frieze pattern classification based on seven symmetry groups.

\section{Wallpaper Pattern Analysis and Generation}

Due to restrictions of weave structure, a woven motif cannot contain rotation in 60 and 120 degrees. This means that the woven pattern cannot contain rotation order of 3- and 6-fold. Therefore, five symmetry groups of wallpaper group cannot be applied, there are only 12 possible symmetry groups which can be applied for woven pattern decoration. This limitation is illustrated by Milasius, Neverauskiene, Katunski, and Kazlauskiene (2002). Since the combination of symmetry operations in the wallpaper group is more complicated than in the Frieze group, so this paper separates symmetric structures of the wallpaper group into three categories based on number of rotation order that contained in the groups, and then constructed three related diagrams. The first diagram is for 1 -fold rotation order where there are totally four symmetry groups in this case, they are $\mathrm{cm}, \mathrm{pm}$, $p g$, and $p 1$. The diagram is illustrated in Figure 2.

The second diagram is for 2-fold rotation order that includes five symmetry groups in the diagram, such as pmm, cmm, pmg, pgg, and $p 2$. Figure 3 illustrates the diagram.

The last diagram is for 4-fold rotation order that consists of only three symmetry groups, namely, $p 4, p 4 m$, and $p 4 g$. It is presented in Figure 4. Because the most traditional motifs are quite big and some of them are symmetric, the wallpaper group cannot apply to them. As a result, the most found symmetry groups of wallpaper patterns on the samples were applied to small and medium motifs. Unfortunately, there are only four of 12 symmetry groups found on the samples namely $\mathrm{pmm}, \mathrm{cmm}$, pm, and $\mathrm{pl}$. 


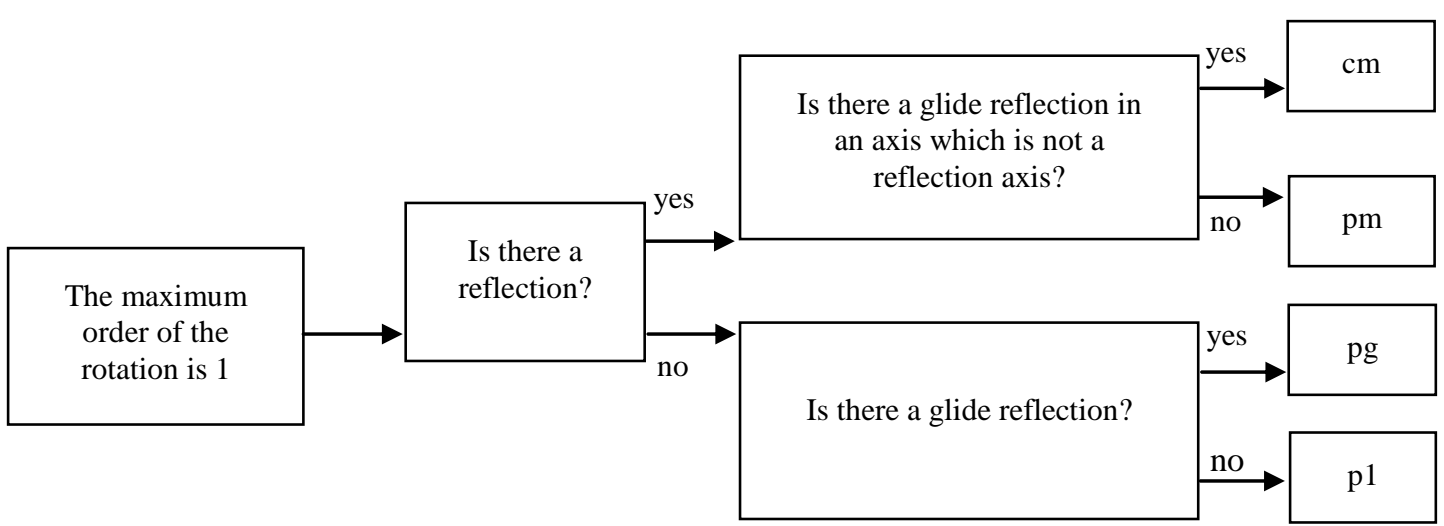

Figure 2. A diagram for wallpaper pattern classification when the maximum order of the rotations equals to one.

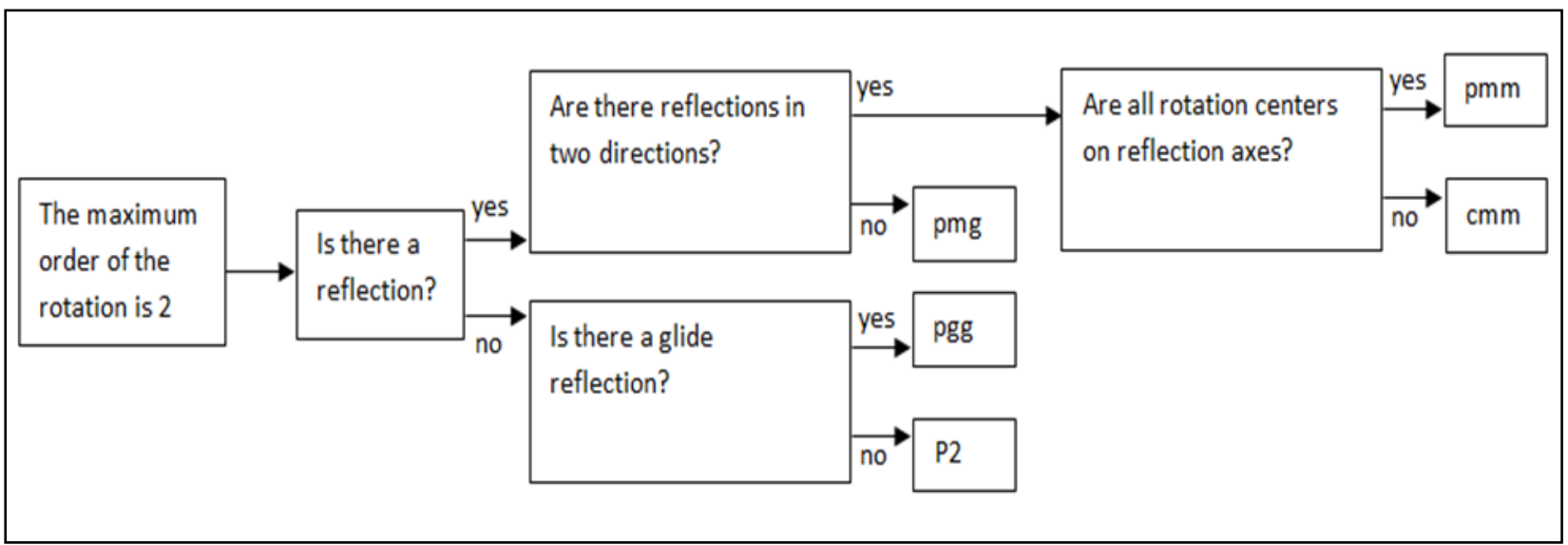

Figure 3. A diagram for wallpaper pattern classification when the maximum order of the rotations equals to two.

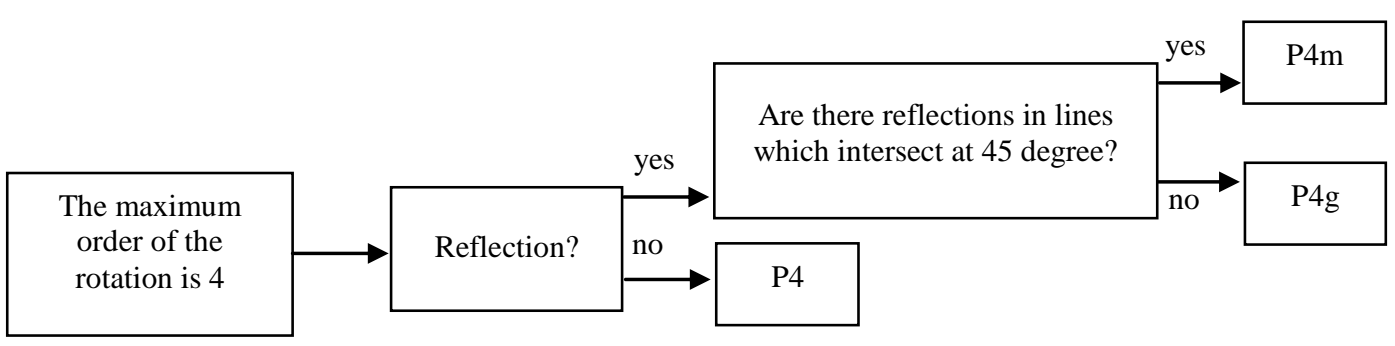

Figure 4. A diagram for wallpaper pattern classification when the maximum order of the rotations equals to four.

The analysis helps to find out common symmetry groups on traditional Lao textiles, it helps to understand characteristics and design styles of Lao textiles. The result will be data to support developing design technique, the implementation of symmetry operations, the symmetry groups of the Frieze and Wallpaper groups see in Chidtavong, Winckler, and Bock (2013) and Chidtavong, Winckler, Bock, and Ellwanger-Mohr (2016). Moreover, the symmetry groups of the Frieze and Wallpaper groups introduce more design styles to the Lao weavers for generating a variety of patterns. In the following section we will explain mathematical approaches for digital representation of Lao textile. 


\section{Digital Representation of Lao Textile}

Generally, a structure of woven textile is created by interlacement of two sets of threads: set of vertical threads called warps and another set of horizontal threads called wefts, regard to this characteristic the information on weaving structure is represented by a binary matrix. Weaving values assign to matrix's elements are modified from Milasius, Neverauskiene, Katunski, and Kazlauskiene (2002). Our study focuses on traditional Lao textile which forms a pattern by weft-faced patterning. Thus, the values of matrix's elements are defined to be true (or number 1) if the interlacement is warp threads under weft threads, the values are false (or number 0) if the interlacement is warp threads over weft threads. Simultaneously, a graphical feature of the weaving structure is represented by a group of binarization squares, the squares interpret as intersection points between warps and wefts while the color of squares depends on the interlacement, if warp threads over weft threads then the color is warp's color, and otherwise the color is weft's color. Moreover, the woven textile has a restriction on rows and columns, every single row and every single column must contain at least one intersection point, this means each row and each column of a binary matrix must have at least one true element. To represent a woven textile in mathematics is defined as below:

$M m \times n$ is a binary matrix of a woven textile, $m$ is number of rows and $n$ is number of columns.

Mix $j$ is a matrix element, $i$ is a row index and $j$ is a column index.

The values of matrix's elements are:

$$
m_{i, j}=\left\{\begin{array}{l}
o, \text { if colof of square }(i, j) \text { is warp's color } \\
1, \text { if color of square }(i, j) \text { is weft's color }
\end{array}\right.
$$

Additional investigated method for pattern generation is based on the advantage of the similarity between treadle loom and Lao floor-loom. Weave components on treadle loom are applied to construct a pattern-draft for Lao floor-loom, the four components of the treadle loom consists are: threading, tie-up, treadling and pattern. This method gives benefit to both weaving on treadle loom and weaving on Lao floor-loom, it facilitates to design a pattern and to modify a pattern as well. Therefore, binary matrices are used to define threading, tie-up, treadling and pattern where the pattern matrix is a result from multiplication of other three binary matrices. The related study of this method can find in Željko, Tomislav, and Danko (2009). The mathematical notations and mathematics equation of the method are illustrated as below:

$$
P p \times f=T n \times f * \operatorname{In} \times m T * H m \times p
$$

$P p \times f$ denotes a Pattern matrix, with $p$ warp thread and $f$ floating supplementary weft.

$T n \times f$ denotes a Treadling matrix, $n$ indicates number of treadle and $f$ indicates number of floating supplementary weft.

I $m \times n$ denotes a tie-up matrix, $m$ indicates number of shaft and $n$ indicates number of treadle.

In $\times m T$ denotes a transpose matrix of tie-up matrix.

$H m \times p$ denotes a Threading matrix, $m$ indicates number of shaft and $p$ indicates number of warp thread.

Due to the fact that binary matrices represent components of weave structure, each row and each column of each matrix must contain at least one true value. On threading matrix, number of column means number of warp on the loom, so each column must contain only one true value that means only one warp tied to one shaft. On treadling matrix, each row contains only one true value, this means that there is only one treadle pressed or only one wooden line picked in each weaving step. For the tie-up matrix, its true values in each column interpret the tying point of shafts to each treadle. An example of pattern generation by this method was 
illustrated in Figure 5, a. is graphical feature of the Tie-up matrix, b. is a graphical feature of Treadling matrix while c. and d. are graphical features of Threading and Pattern matrices respectively. The black square on tie-up, Threading and Treadling matrices indicate true value while a white a square indicates false value while the color on the pattern matrix depends on warp's color and weft's color. The mathematical equations (1) and (2) are implemented for generating two standards weave files that will be explained as follow.

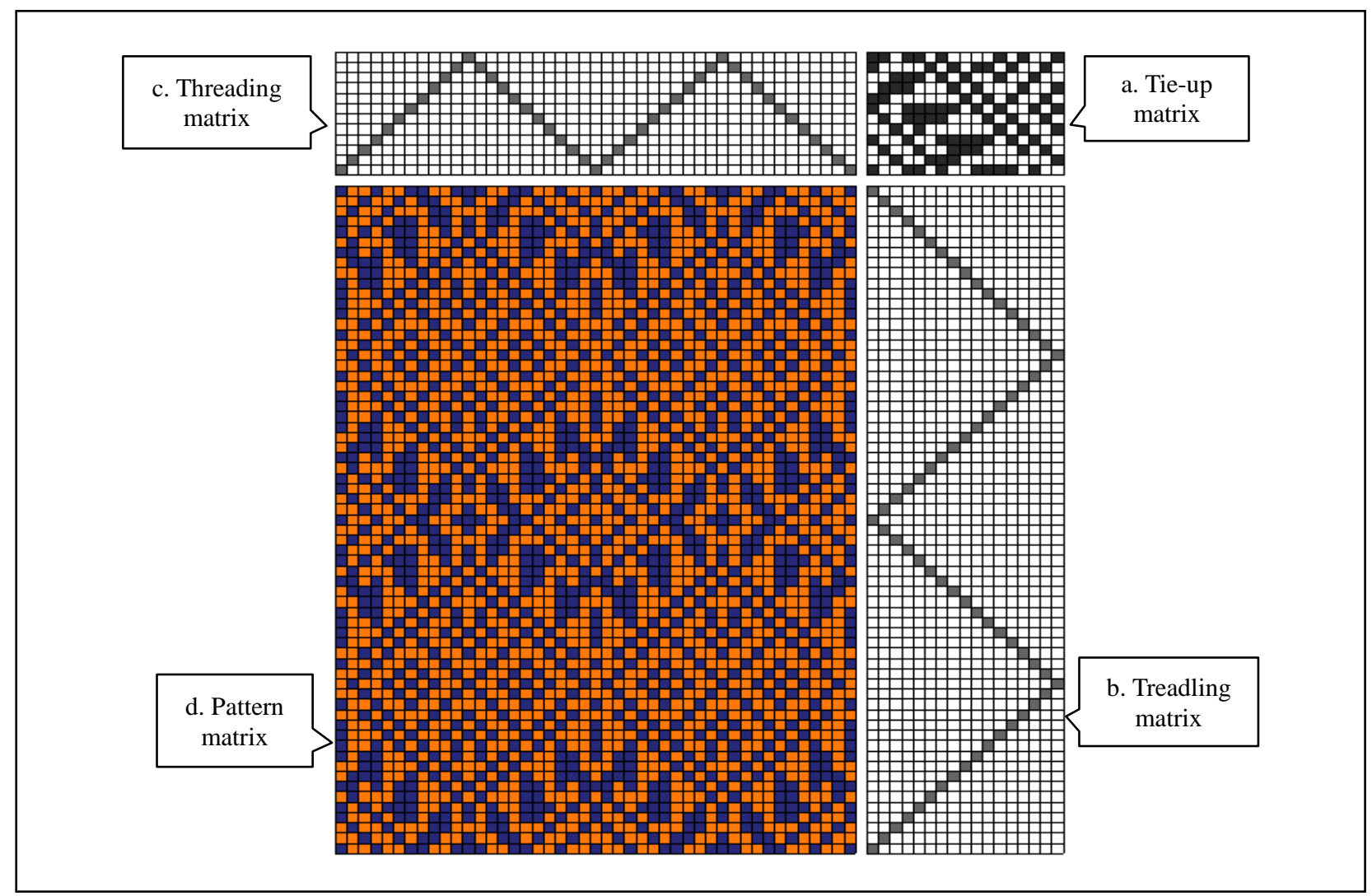

Figure 5. A graphical representation of weave components.

\section{Standard Weave File for Digital Weave-Draft}

As mentioned in the beginning, this paper will present two standard weave files for representing digital weave-draft, namely: image file and WIF file. The digital weave-drafts are intended to be understandable for hand-weavers and electronic looms. The WIF file developed based on weave structure of Dobby hand floor-loom (Roberts, 1912) and it is created by implementing equation (2) and it is followed WIF specification (WIF Specification, 1997) as well. Due to the fact that a pattern is generated by multiplication of three binary matrices, Tie-up, Threading, and Treadling matrices, so instead of directly storing binary data of a pattern, WIF stores information of these three binary matrices. According to WIF specification, WIF file consists of a set of sections and key names, the section names are in brackets such as, "Weaving", "WARP", and "WEFT" sections. The key names are followed by an equal sign and the data, such as: shafts=16 and treadles=16. The sections are defined as "INFORMATIONAL" and "DATA" sections. For example, "WIF" and "CONTENTS" sections are informational section, where the "CONTENTS" section is a special informational section that lists all other included sections. Dimension and binary data of Tie-up, Threading, and Treadling matrices are stored in specific data sections, such as number of shafts and number of treadles can be found in "WEAVING" section, 
number of warp threads is stored in "WARP" section, and number of weft threads is stored in "WEFT" section. Binary data for Tie-up matrix are listed in "TIEUP" section, number on the left equal sign indicates column's index while a sequence number on the right equal sign is a list of row's indexes on that column; "THREADING" section is listed binary data for Threading matrix, number on the left equal sign indicate column's index while a number on the right equal sign is row's index; and "TREADLING" section is listed binary data of Treadling matrix, number on the left equal sign indicates row's index while a number on the right equal sign is column's index. Instead of storing all binary data of matrices, WIF file is stored only row's index or column's index that has true value where the row's index or column's index on the right equal sign indicates row or column of matrix that has true value. By reading data in these sections from a given WIF file and work together with equation (2), it is able to generate a pattern matrix. For more detail on WIF structure see WIF Specification (1997).

The image file is a binary image that contains only two colors, file extension can be .jpg, .tiff, .bpm, etc. One color indicates warp thread and another color indicates weft thread. A binary image of woven pattern actually is a pattern matrix in equation (2). The next section will show weaving experiments with sample digital weave-drafts on TC2 loom and explain their results.

\section{Weaving Lao Style on TC2 Loom}

Due to the fact that weaving machines work with digital drafts, the digital weave-drafts in both formats, image and WIF files are input for weaving on electronic looms. The electronic looms actually are able to weave many kinds of fabrics, the complex on the weave structures depends on their given digital weave-drafts. Generally the electronic looms read only binary data on the given drafts and the drafts are read row by row in order to make interlacement between warps and wefts. Therefore, to weave textiles like traditional Lao style on the electronic looms is not a big deal if we know their weaving techniques and use the suitable looms. As a result, this study uses a modern TC2 loom for testing our digital weave-drafts. The TC2 loom is a hand operated loom which allows weavers to have complete control over warp threads, it provides weave technique like weaving on Lao floor-loom. Digital weave-drafts are generated based on characteristics of Lao textiles and two considering points of Lao weaving technique. The first point is that traditional Lao weaving technique is always used two weave structures, a ground-weave and a pattern-weave. It means, we need a weave-draft to tell a machine to weave this combination structure. The second is that the most Lao textiles are colorful fabrics which are used more than one weft for pattern-weave, so weavers have to define how many wefts are intended to use during weaving. Figure 6 shows a digital weave-draft of Siho motif with size $660 \times 557$, Figure 7 shows weaving result of the given weave-draft.

The weaving results show compatibility between the digital weave-drafts and the electronic TC2 loom. The results show a variety of colorful motifs and patterns on a fabric. Because the TC2 loom is a hand operated loom, it provides various possibilities to decorate fabric; this property satisfies the characteristics of Lao textiles. The experiment focuses on weaving style of traditional Lao textile which consists of pattern- and ground-weave (plain weave). Therefore, a hand-pick decoration is used to weave a complicated pattern. The decoration is up to imagination of the designer. The produced fabrics look the same like fabrics that are woven on Lao floor-loom. Therefore, the results express a connection between traditional style of Lao textiles and modern weave technology. They fulfill the gap between traditional weave technique of Lao weavers and modern weave technique. For more details on weave result see Chidtavong, Winckler, Bock, and Ellwanger-Mohr (2016). 


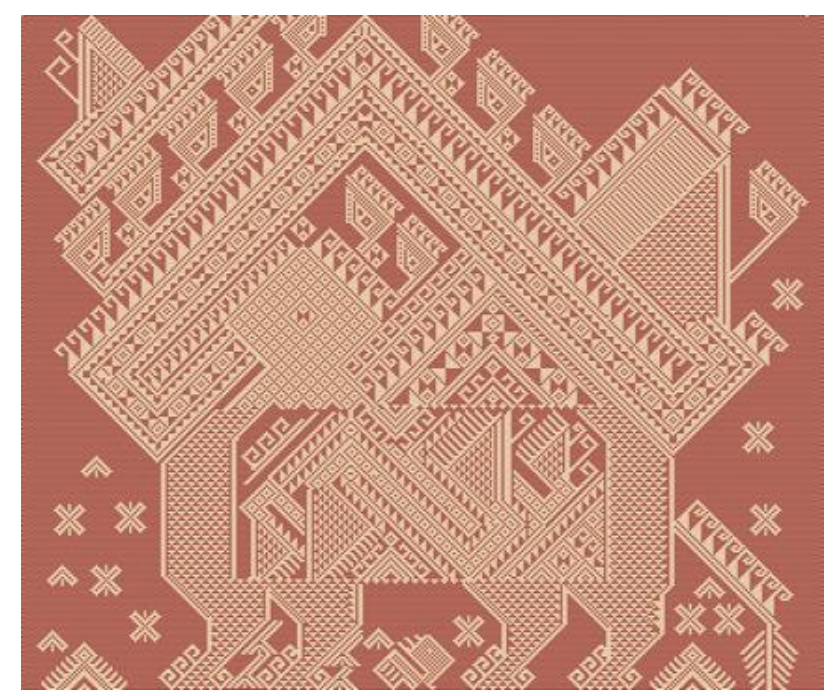

Figure 6. A digital weave-draft of the Siho motif (size: $660 \times 557$ ).

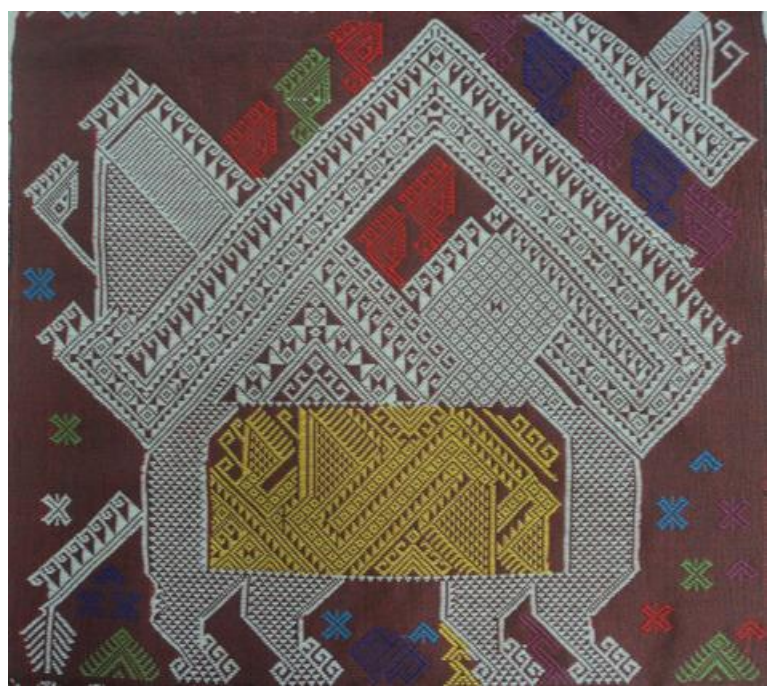

Figure 7. Weaving result of the weave-draft of Siho motif on TC2 loom.

\section{Conclusion}

This paper presents scientific methods for digitizing motifs, patterns, and weave-drafts for traditional Lao textiles. The seven symmetry groups of Frieze group and 12 symmetry groups of the Wallpaper group are introduced for analyzing sample of ethnic patterns and for generating woven pattern in design task. The study found that digital weave-draft is a key to connect between traditional weave techniques to modern weave techniques. Therefore, the mathematical equations for two standard weave files are explained, the files are used as digital weave-draft for electronic loom. The digital weave-drafts are tested with a modern TC2 loom, the results show a connection between traditional style of Lao textiles and modern weave technology.

\section{References}

Schattschneider, D. (1978). The plane symmetry group: Recognition and notation. American Mathematical Monthly, 85, 439-450. Chidtavong, L., Winckler, M. J., \& Bock, H. G. (2013). Tying up the Naga: Motif design for Lao textile. Proceeding from 4th SCCH, Heidelberg Academy of Sciences and Humanities. 
Chidtavong, L., Winckler, M. J., Bock, H. G., \& Ellwanger-Mohr, M. (2016). Analysis, modeling and generation of traditional Lao woven textile. Retrieved from Digital Dissertation: http://archiv.ub.uni-heidelberg.de/volltextserver/20605/

Radaelli, P. (2011). Symmetry in crystallography: Understanding the international tables. Oxford Scholarship.

Roberts, T. (1912). Tappet and Dobby looms: Their mechanisms and management. Manchester, EMMOTT \& Co., Limited, 65 King Street. London: 20 Bedford Street, W.C.

Milasius, V., Neverauskiene, D., Katunski, J., \& Kazlauskiene, I. (2002). The mathematical basis of ornamentation of patterned woven fabrics. Fibres \& Textiles in Eastern Europe.

Vestby, V. (1990). Digital Weaving Norway (DWN). Thread Controller 2 (TC2)-digital weaving looms. Retrieved from http://www.digitalweaving.no/en/

WIF Specification. (1997). Retrieved from http://www.mhsoft.com/wif/wif1-1.txt

Liu, Y., \& Collins, R. T. (1998). Frieze and wallpaper symmetry groups classification under affine and perspective distortion. The Robotics Institute Carnegie Mellon University.

Željko, P., Tomislav, S., \& Danko, B. (2009). Computer aided construction of reinforced weaves using matrix calculus. Fibres \& Textiles in Eastern Europe, 17(5), 43-48. 